CERN-TH.7473/94

hep-th/9410104

\title{
SPACE TIME INTERPRETATION OF S-DUALITY AND SUPERSYMMETRY VIOLATIONS OF T-DUALITY
}

\author{
Ioannis Bakas 周 \\ Theory Division, CERN \\ CH-1211 Geneva 23, Switzerland
}

\begin{abstract}
The S-duality transformations of the lowest order string effective theory admit a space time interpretation for 4-dim backgrounds with one Killing symmetry. Starting from pure gravity and performing a sequence of intertwined $\mathrm{T}-\mathrm{S}-\mathrm{T}$ duality transformations we obtain new solutions which are always pure gravitational. In this fashion, S-duality induces an $S L(2, R)$ transformation in the space of target space metrics which coincides with the action of the Ehlers-Geroch group and interchanges the electric with the magnetic aspects of gravity. Specializing to gravitational instanton backgrounds we show that ALE instantons are mapped to (multi) Taub-NUT backgrounds and viceversa. We find, however, that the self-duality of the metric is not generically preserved, unless the corresponding Killing vector field has self-dual covariant derivatives. Thus, the T-S-T transformations are not always compatible with the world-sheet supersymmetry of $N=4$ superconformal string vacua. We also provide an algebraic characterization of the corresponding obstruction and associate it with a breakdown of space time supersymmetry under rotational $\mathrm{T}$-duality transformations.
\end{abstract}

CERN-TH.7473/94

October 1994

*Permanent address: Department of Physics, University of Crete, GR-71409 Heraklion, Greece

${ }^{\dagger} \mathrm{e}-$-mail address: BAKAS@SURYA11.CERN.CH 
The toroidal compactification of the heterotic string to $M_{4} \times T^{6}$ seems to exhibit a remarkable symmetry under an $S L(2, Z)$ group of transformations which act on the coupling constant of the theory [1,2]. This discrete symmetry, known as S-duality, has its origin in the lowest order 4-dim effective theory where the axion-dilaton system admits a continuous $S L(2, R)$ symmetry that becomes manifest in the Einstein frame. The purpose of this paper is to show that $\mathrm{S}$-duality can have a space time interpretation when it is appropriately combined with $\mathrm{T}$-duality associated to a Killing symmetry on $M_{4}$. If both symmetries are valid and remain consistent with supersymmetry, then different space time backgrounds of the heterotic string will be related. We will see, for instance, that in the toroidal compactification of the heterotic string, S-duality relates vacua with $M_{4}$ being either the flat or the Taub-NUT space for certain discrete values of its moduli parameter. It is not surprising that both these spaces admit four standard space time supersymmetries [3].

We consider bosonic backgrounds which are supersymmetric solutions of the heterotic string and so all fermion fields together with their supersymmetric variations are taken zero. As for the gauge fields, the standard embedding is implicitly used, in order to insure that all higher order $\alpha^{\prime}$ corrections which are proportional to $\operatorname{Tr} R \wedge R-\operatorname{Tr} F \wedge F$ vanish. The known bosonic solutions of the heterotic string correspond to supersymmetric self-dual backgrounds for which the standard embedding of the gauge fields $A$ amounts to the self-duality condition on $F(A)[4-6]$. We use this as a strarting point to show that if a self-dual background admits Killing symmetries, its $\mathrm{T}$-dual will not necessarily be supersymmetric and hence it will not always qualify for a new bosonic solution of the heterotic superstring. As we will see later, there are two types of Killing vector fields distinguished by the requirement of preserving standard supersymmetry or not. The first type, called translational, has self-dual covariant derivatives and preserves the vanishing condition of the supersymmetric variations of the fermion fields. There exist, however, rotational Killing vector fields, in that their covariant derivatives are not self-dual, which are not compatible with space time supersymmetry. The ordinary gravitational instantons (including flat space) always admit at least one translational Killing symmetry and so their T-dual versions are legitimate solutions. But if one chooses a rotational Killing symmetry, which is present in simple examples like the flat space and the Eguchi-Hanson instanton, then a classical supersymmetric anomaly appers by performing the corresponding T-duality transformation. This result casts doubts on the string equivalence of $\mathrm{T}$-duality related backgrounds and calls for a careful investigation in the general supersymmetric case [7].

Most of the present work concentrates on the properties and symmetries of the lowest order 4-dim effective bosonic theory [8],

$$
S_{e f f}=\int_{M_{4}} d^{4} X \sqrt{\operatorname{det} G} e^{-2 \Phi}\left(R[G]+4(\nabla \Phi)^{2}-\frac{1}{12} H^{2}\right)
$$

after supressing the contribution of the gauge fields. Here $H_{\mu \nu \rho}=3 \nabla_{[\mu} B_{\nu \rho]}$ is the field strength of the anti-symmetric tensor field $B_{\mu \nu}$ and $\Phi$ is the dilaton field. The cosmological constant (central charge deficit) is also taken to be zero in the lowest order effective 
action. From now on, we will consider the general class of 4-dim metrics with (at least) one Killing symmetry associated to a vector $K=\partial / \partial \tau$ on $M_{4}$. Then, it is well known that any such metric can be written locally in the form

$$
d s^{2}=V\left(d \tau+\omega_{i} d x^{i}\right)^{2}+V^{-1} \gamma_{i j} d x^{i} d x^{j}
$$

where $\left\{x^{i} ; i=1,2,3\right\}$ are coordinates on the space of non-trivial orbits of $\partial / \partial \tau$ in $M_{4}$ and $V, \omega_{i}, \gamma_{i j}$ are all independent of $\tau$, but otherwise arbitrary. The results we will describe are quite general, but some of their implications will be considered in the context of the heterotic string theory.

The solutions of the vacuum Einstein equations can be regarded as special cases of gravitational string backgrounds with zero dilaton $\Phi$ and anti-symmetric tensor field $B_{\mu \nu}$. In view of this embedding, if we perform a $\mathrm{T}$-duality transformation (see for instance [9] and references therein)

$$
\begin{aligned}
\tilde{G}_{\tau \tau} & =\frac{1}{G_{\tau \tau}}, \quad \tilde{G}_{\tau i}=\frac{B_{\tau i}}{G_{\tau \tau}}, \quad \tilde{G}_{i j}=G_{i j}-\frac{G_{\tau i} G_{\tau j}-B_{\tau i} B_{\tau j}}{G_{\tau \tau}} \\
\tilde{B}_{\tau i} & =\frac{G_{\tau i}}{G_{\tau \tau}}, \quad \tilde{B}_{i j}=B_{i j}-\frac{G_{\tau i} B_{\tau j}-G_{\tau j} B_{\tau i}}{G_{\tau \tau}} \\
\tilde{\Phi} & =\Phi-\frac{1}{2} \log G_{\tau \tau}
\end{aligned}
$$

to the pure gravitational metric (2), new solutions to the string background equations will result with non-trivial $\Phi$ and $B_{\mu \nu}$ fields in general.

On the other hand, the effective theory (1) exhibits an additional global $S L(2, R)$ symmetry which is manifestly described in terms of the axion-dilaton system formulated in the Einstein frame of the string,

$$
G_{\mu \nu}^{(E)}=e^{-2 \Phi} G_{\mu \nu}
$$

In this frame, the axion field $b$ can be consistently defined as

$$
\partial_{\mu} b=\frac{e^{-4 \Phi}}{6} \sqrt{\operatorname{det} G^{(E)}} \epsilon_{\mu}{ }^{\nu \rho \sigma} H_{\nu \rho \sigma}
$$

where $\epsilon_{\tau 123}=1$, and so $b$ is non-locally related to the original variables. The axiondilaton system behaves in the Einstein frame as an $S L(2, R) / U(1)$ non-linear $\sigma$-model, which in terms of the pair of conjugate variables

$$
S_{ \pm}=b \pm e^{-2 \Phi}
$$

remains invariant under the $S L(2, R)$ group of transformations

$$
S_{ \pm} \rightarrow \frac{A S_{ \pm}+B}{C S_{ \pm}+D}, \quad A D-B C=1 .
$$

We will refer to this symmetry of the 4-dim effective theory as S-duality, although it is appropriate to reserve the name duality for discrete string symmetries and not just for 
continuous symmetries of the lowest order effective theory. The $S L(2, Z)$ string duality symmetry will be considered later, when additional restrictions will be also imposed on the background metric.

The main observation is that by performing a sequence of $\mathrm{T}-\mathrm{S}-\mathrm{T}$ duality tranformations on a pure gravitational background with one Killing symmetry, the resulting string background is pure gravitational as well. Since the loop of T-S-T operations turns out to be a generic $S L(2, R)$ transformation in the space of target space metrics, it can be regarded as providing a space time interpretation of S-duality. Moreover, as we are going to show, this $S L(2, R)$ transformation in the space of Ricci flat metrics coincides with the action of the Ehlers-Geroch symmetry in ordinary general relativity upon reduction from four to three dimensions $[10,11]$. Therefore, thinking of ordinary gravity as a special case of the 4-dim string background equations, we can provide an alternative description of the Ehlers-Geroch symmetry as being induced by S-duality by employing suitably $\mathrm{T}$-duality on the way, switching on and off non-trivial axion and dilaton fields. Another advantage of this interpretation that will be discussed later, is that $\mathrm{S}$-duality can be consequently understood as a symmetry interchanging the electric with the magnetic aspects of gravity, since to each metric of the form (2) there is associated a natural Maxwell field,

$$
A=V\left(d \tau+\omega_{i} d x^{i}\right)
$$

We proceed by performing first the T-duality transformation (3)-(5) to the metric (2) within the string effective theory (1). The result of the calculation in the Einstein frame of the string can be summarized as follows,

$$
\tilde{G}_{\mu \nu}^{(E)}=\left(\begin{array}{cccc}
1 & 0 & 0 & 0 \\
0 & & & \\
0 & & \gamma_{i j} & \\
0 & & &
\end{array}\right)
$$

and

$$
\tilde{\Phi}=-\frac{1}{2} \log V, \quad \tilde{B}_{\tau i}=\omega_{i},
$$

while all other components are zero. Of course $\omega_{i}$ (and hence $\tilde{B}_{\tau i}$ ) are defined up to a gauge transformation

$$
\omega_{i} \rightarrow \omega_{i}-\frac{\partial \lambda}{\partial x^{i}}
$$

which amounts to the coordinate (shift) transformation

$$
\tau \rightarrow \tau+\lambda\left(x^{i}\right)
$$

Using the defining relations for the axion field $b$, we find in this case the result

$$
\partial_{i} b=\frac{1}{2} V^{2} \sqrt{\operatorname{det} \gamma} \epsilon_{i}^{j k}\left(\partial_{j} \omega_{k}-\partial_{k} \omega_{j}\right)
$$


since $\epsilon_{\tau i j k}=\epsilon_{i j k}$ in the metric (11). In the terminology of Gibbons and Hawking [11], $b$ coincides with the so called nut potential of the metric (2). The nut charge of a gravitational solution can be interpreted as the axion charge of the $\mathrm{T}$-dual string solution. To perform the S-duality on the resulting axion-dilaton system we consider the corresponding pair of conjugate fields

$$
S_{ \pm}=b \pm e^{-2 \Phi}=b \pm V
$$

It can be easily verified that the $S L(2, R)$ transformation (9) reads for the $V$ and $b$ fields as

$$
\begin{gathered}
V^{\prime}=\frac{V}{(C b+D)^{2}-C^{2} V^{2}}, \\
b^{\prime}=\frac{(A D+B C) b+A C\left(b^{2}-V^{2}\right)+B D}{(C b+D)^{2}-C^{2} V^{2}},
\end{gathered}
$$

while the 3-metric $\gamma_{i j}$ remains invariant. After this transformation, the new axion field can be written again in the form (15) using the resulting expression (17) for the new $V^{\prime}$ and assuming the existence of a new vector field $\omega_{i}^{\prime}$. Hence, applying again a T-duality transformation, it is obvious that closing the loop of $\mathrm{T}-\mathrm{S}-\mathrm{T}$ operations yields a pure gravitational background of the form (2) for the primed variables $V^{\prime}$ and $\omega_{i}^{\prime}$, but with the same $\gamma_{i j}$. The computation shows that $\omega_{i}^{\prime}$ is only implicitly determined by $V$ and the nut potential $b$ of the original metric in terms of the coupled system of equations

$$
\frac{1}{2} \sqrt{\operatorname{det} \gamma} \epsilon_{i}^{j k}\left(\partial_{j} \omega_{k}^{\prime}-\partial_{k} \omega_{j}^{\prime}\right)=\frac{1}{V^{2}}\left(\left((C b+D)^{2}+C^{2} V^{2}\right) \partial_{i} b-2 C V(C b+D) \partial_{i} V\right)
$$

Clearly, $\omega_{i}^{\prime}$ are non-local expressions of the original data, but it is important for our purposes that they exist and they are unique, up to gauge transformations of the form (13).

The result we have just described, obvious as it may seem in retrospect, provides a space time interpretation of S-duality, which in the present context coincides with the Ehlers-Geroch $S L(2, R)$ symmetry group of vacuum Einstein spaces with (at least) one Killing symmetry. To make the identification exact, note that group elements of the form $C=0, A=D=1$ shift $b$ by a constant $B$ and keep $V$ invariant (translations), while group elements of the form $B=C=0, D=A^{-1}$ scale both $V$ and $b$ by a constant $A^{2}$ (dilations). The really non-trivial part of the symmetry is described by the group element with $A=D=1$ and $B=0$, which is nothing else but the celebrated Ehlers transform of the 4-dim metric. It is also interesting to note that from this point of view, the three parameter group of transformations (9) describes a duality between the electric aspects of gravity, characterized by $V$, and the magnetic aspects characterized by the nut potential $b$ (being the axion field in the $\mathrm{T}$-dual string setting of the problem). These electric and magnetic aspects of gravity can be best understood by computing the components of the curvature of the Maxwell field (10) associated with any metric of the form (2). Hence, S-duality can be regarded once more as being analogous to the well known duality symmetry of electromagnetism. 
We are considering 4-dim space times with one Killing symmetry and so the isometry group that is generated by the vector field $\partial / \partial \tau$ might have fixed points in its action. It is known that the corresponding fixed points are either isolated (called nuts) or they form 2-dim submanifolds (called bolts). Bolts are thought to be analogous to electric type mass-monopoles, while nuts as gravitational dyons with real electric mass-monopole and imaginary magnetic mass-monopole [11]. The existence of nuts and bolts in Euclidean gravity and the relation between them is a manifestation of the space time interpretation of S-duality we have presented. Single nuts are not physically acceptable in classical relativity, but bolts are, because of the asymptotic behaviour of the corresponding solutions. However, if a string theory had $S L(2, Z)$ S-duality as an exact symmetry, then roughly speaking there would be an equivalence mapping between nuts and bolts in that string framework. We will make this idea concrete next in the context of $N=4$ superconformal string theory, where S-duality can relate asymptotically locally Euclidean (ALE) gravitational instanton backgrounds to (multi) Taub-Nut backgrounds, and vice-versa.

It is an established result in the literature that for pure gravitational backgrounds, $N=4$ supersymmetry on the world-sheet implies the hyper-Kahler condition on the metric. In four dimensions this means that Ricci flat metrics are self-dual (or anti selfdual), i.e.,

$$
R_{\mu \nu \rho \sigma}= \pm \frac{1}{2} \sqrt{\operatorname{det} G} \epsilon_{\rho \sigma}^{\kappa \lambda} R_{\mu \nu \kappa \lambda}
$$

and so they qualify as gravitational instanton backgrounds. We will consider the selfdual case picking up the plus sign, while the anti self-dual case can be treated in a similar way. One expects that if $\mathrm{T}$-duality always preserves space time supersymmetry in $N=4$ superconformal string theory, the application of a $\mathrm{T}-\mathrm{S}-\mathrm{T}$ sequence of operations on self-dual metrics with one Killing symmetry will preserve self-duality and provide an S-duality relation between gravitational instanton backgrounds. In the heterotic string theory it is sufficient to consider the lowest order effective action, since all higher order corrections in $\alpha^{\prime}$ can be made zero by the standard embedding of the gauge fields. Also, it is sufficient for our argument to consider only the bosonic sector of the theory and reexamine the action of the Ehlers-Geroch group on self-dual metrics. It will turn out that some surprises arise for a certain class of metrics satisfying various additional technical conditions, while testing the T-S-T duality transformations. We will also show that for self-dual backgrounds of ALE or Taub-Nut type the self-duality condition can be preserved by the transformation, but again not always.

Self-dual metrics of the form (2) can be classified into two classes depending on the type of the Killing symmetry. In particular, there are two possibilities depending on whether the covariant derivative of the Killing vector field $K_{\nu}, \nabla_{\mu} K_{\nu}$, is self-dual or not [12]. The algebraic condition that leads to this distinction is solely described by the characteristic quantity

$$
\Delta S_{-} \equiv \gamma^{i j} \partial_{i} S_{-} \partial_{j} S_{-}
$$

where $S_{-}$is one of the two conjugate variables (8) involving the nut potential $b$ (equivalently the axion in the $\mathrm{T}$-dual formulation) and $V$ of the original metric. Since the 
3-metric $\gamma_{i j}$ has Euclidean signature +++ , the quantity $\Delta S_{-}$is non-negative. The two different cases in question arise when $\Delta S_{-}=0$ or $\Delta S_{-}>0$. . In the first case the Killing symmetry is usually called "translational", while in the second case "rotational", although the real distiction between the two depends on the quantity (21) being zero or strictly positive. Of course, it might be possible for a self-dual metric to have more than one Killing symmetry with respect to which is either of one or of the other type; the Eguchi-Hanson instanton is the simplest non-trivial example with two such different types of Killing symmetries, but the flat space can entertain both possibilities as well. It is important to realize that the analysis we will present in the sequel depends crucially on the Killing symmetry that is chosen to perform the T-S-T transformation.

In the first case, when $\Delta S_{-}=0$ and hence $S_{-}$is constant, there is a theorem [12, 13] stating that there exists a coordinate system in which $\gamma_{i j}=\delta_{i j}$ and the self-duality condition amounts to the condition

$$
\partial_{i} V^{-1}=\frac{1}{2} \epsilon_{i j k}\left(\partial_{j} \omega_{k}-\partial_{k} \omega_{j}\right),
$$

with respect to the flat 3-metric $\delta_{i j}$. Hence, $V^{-1}$ satisfies the 3-dim Laplace equation and all components of the 4-dim metric are determined in terms of its solutions. In this case, comparing eqs. (15) and (22), we find that

$$
S_{+}=2 V, \quad S_{-}=0,
$$

up to an overall constant which is taken to be zero with no loss of generality. Solutions to the 4-dim string background equations, where a (conformally) flat metric is coupled to axionic instantons have been considered before $[4,5]$, together with their $\mathrm{T}$-dual formulations as pure gravitational instanton backgrounds [6]. The main issue of our concern is not to rederive this result, but to note that the sequence of operations T-S-T preserves self-duality and the characteristic condition $\Delta S_{-}=0$. Since the only nontrivial part of the Ehlers-Geroch symmetry corresponds to the choice $B=0, A=D=1$, applying formula (17) we find the effect of the Ehlers transformation to be

$$
V^{-1} \rightarrow V^{-1}+2 C,
$$

where in the discrete $S L(2, Z)$ case $C$ is an arbitrary integer. Its effect on $\omega_{i}$ is trivial and up to gauge transformations we may take $\omega_{i}^{\prime}=\omega_{i}$. This shift is clearly a symmetry of the 3-dim Laplacian equation imposed on $V^{-1}$ by the self-duality and although it is rather simple, it can have important effects on the space time interpretation of the corresponding solutions.

*If we were considering anti self-dual metrics then there would be an analogous condition on $S_{+}$ instead, which in more geometrical terms would be equivalent to having an anti self-dual $\nabla_{\mu} K_{\nu}$ or not. The reason is that if a metric of the form (2) is self-dual, its anti self-dual counterpart will be obtained by flipping the sign of all $\omega_{i}$ and hence of the corresponding $b$. Under this interchange we clearly have $S_{ \pm} \rightarrow-S_{\mp}$. 
The localized solutions of the 3-dim Laplace equation for $V^{-1}$ are of the general form

$$
V^{-1}=\epsilon+\sum_{i=1}^{n} \frac{m_{i}}{\left|\vec{x}-\vec{x}_{0, i}\right|},
$$

modulo delta functions, where $m_{i}$ and $\vec{x}_{0, i}$ are moduli parameters. The constant $\epsilon$ can be either zero or non-zero, in which case it is usually normalized to 1 . If all $m_{i}=M$ and if $\tau$ is periodic with the range $0 \leq \tau \leq 4 \pi M / n$, then it is known that the singularities of the corresponding 4-dim self-dual metric are removable and the solutions could qualify as gravitational instanton backgrounds [11] (see also [14] for a review). From now on, the singularities of the metric, being either nuts or bolts depending on the coordinate system, will be assumed to be removable. For $\epsilon=1$ one has the multi Taub-NUT metrics, with $n=1$ being the ordinary self-dual Taub-NUT metric. For $\epsilon=0$ the resulting metrics are the multi-center Gibbons-Hawking metrics, with $n=2$ being the simplest non-trivial example known as the Eguchi-Hanson instanton. They correspond to the A-series in the A-D-E classification of the ALE gravitational instantons [15]. For $\epsilon=0$ and $n=1$ one obtains the flat metric space by appropriate coordinate transformation. In gravitational theories, only the solutions with $\epsilon=0$ are physically acceptable based on their asymptotically locally Euclidean behaviour; the boundary at infinity is the lens space $S^{3} / Z_{n}$. For the multi Taub-NUT metrics, the existence of a non-zero nut parameter violates the ALE asymptotic condition and the solutions are asymptotically flat only in the spatial direction $|\vec{x}| \rightarrow \infty$, but they are periodic in the variable $\tau$. In other words, $\epsilon$ being zero or not changes the sense in which the solution is asymptotically flat from the four to the three dimensional case.

It is often the case with symmetry groups of differential equations that their action on the space of solutions does not respect boundary conditions. In the space time interpretation we are presently considering, this means that S-duality can in principle relate geometrically (and even topologically) different backgrounds. In particular, under the Ehlers transform ALE instantons are mapped to multi Taub-NUT backgrounds and vice versa, as it was already indicated. This changes the geometry and the asymptotic behaviour of the corresponding solutions. Suppose that we start from a multi-center Gibbons-Hawking instanton and set $M=1$ by rescaling simoultaneously all the space time coordinates. Then, the Ehlers transform of $V^{-1}$ gives rise to a multi Taub-NUT space with $M=1 / 2 C$, provided that $C \neq 0$. The period of $\tau$ scales accordingly under this transformation. Reversely, starting from multi Taub-NUT backgrounds it is possible to obtain the multi-center Gibbons-Hawking instantons. This is more easily seen if $\epsilon$ is not normalized to 1 but it is allowed to assume arbitrary non-zero values; otherwise a composition of the Ehlers transform (24) with the dilations will be necessary to complete the mapping. It is appropriate to consider $C>0$ in all these cases.

The Ehlers transform maps the flat space to the ordinary Taub-NUT space. This case corresponds to self-dual metrics with a single center which can be positioned at the

${ }^{\dagger}$ The string equivalence between geometrically and even topologically different backgrounds which are related by $\mathrm{T}$-duality was considered before [16]. 
origin using the freedom of translations in $\vec{x}$-space. The metric with $\epsilon=0$ has $V=|\vec{x}|$ and

$$
\omega_{1}=-\frac{x^{2} x^{3}}{|\vec{x}|\left(\left(x^{1}\right)^{2}+\left(x^{2}\right)^{2}\right)}, \quad \omega_{2}=\frac{x^{1} x^{3}}{|\vec{x}|\left(\left(x^{1}\right)^{2}+\left(x^{2}\right)^{2}\right)}, \quad \omega_{3}=0 .
$$

If we introduce two complex coordinates $z_{1}$ and $z_{2}$ so that

$$
\begin{aligned}
& x_{1}=\frac{1}{2} \operatorname{Im}\left(\bar{z}_{1} z_{2}\right), \quad x_{2}=\frac{1}{2} \operatorname{Re}\left(\bar{z}_{1} z_{2}\right), \\
& x_{3}=\frac{1}{4}\left(\left|z_{1}\right|^{2}-\left|z_{2}\right|^{2}\right), \quad \tau=\operatorname{Im} \log \left(z_{1} z_{2}\right),
\end{aligned}
$$

the metric assumes the flat form with $z_{1}$ and $z_{2}$ as its corresponding Kahler coordinates. Its Ehlers transform with respect to the Killing vector field $\partial / \partial \tau$ is the Taub-NUT metric. The period of the $\tau$ coordinate is $2 \pi / C$ after removing the singularity. Since both these 4 - $\operatorname{dim}$ backgrounds admit $N=4$ space time supersymmetry [3], it is natural to expect that in the heterotic string theory they will be related by $\mathrm{S}$-duality for discrete values of $C$. This novel possibility has to be tested directly. It is curious to note that the compactified Schwarzschild solution can be regarded as a combination of a self-dual Taub-NUT metric with its anti self-dual partner, in order to cancel the magnetic mass.

We will now examine the other general class of self-dual metrics with one rotational symmetry corresponding to the algebraic condition $\Delta S_{-}>0$. There is a theorem [13] stating that in this case the self-dual metrics are determined by a single scalar function $\Psi\left(x^{i}\right)$ in a coordinate system of the form (2) so that

$$
\gamma_{11}=\gamma_{22}=e^{\Psi}, \quad \gamma_{33}=1
$$

while the off-diagonal components of the 3-metric are zero and

$$
V^{-1}=\partial_{3} \Psi, \quad \omega_{1}=-\partial_{2} \Psi, \quad \omega_{2}=\partial_{1} \Psi, \quad \omega_{3}=0 .
$$

Then, in terms of this parametrization the self-duality condition for the 4 -metric becomes the continual Toda equation for $\Psi$, namely

$$
\left(\partial_{1}^{2}+\partial_{2}^{2}\right) \Psi+\partial_{3}^{2} e^{\Psi}=0
$$

Performing the $\mathrm{T}$-duality on such a gravitational background we find that

$$
S_{+}=2 V-x^{3}, \quad S_{-}=-x^{3},
$$

up to an overall constant and with Einstein metric given by eq. (11). In this coordinate system $\Delta S_{-}$remains positive (as it should be for a coordinate independent description of the characteristic condition) and takes the value 1 .

It is interesting to note at this point that the space of solutions of the continual Toda equation exhibits infinitely many symmetries generating a classical $W_{\infty}$ algebra [17]. The simplest representative (associated with the centerless Virasoro algebra) is given infinitesimally by the transformation

$$
\delta \Psi=\partial \epsilon+\epsilon \partial \Psi
$$


where the infinitesimal parameter $\epsilon$ depends on the chiral combination $2 z=x^{1}+i x^{2}$ and the derivatives are taken with respect to $z$. There is a similar transformation for the other chiral sector in terms of $2 \bar{z}=x^{1}-i x^{2}$. Higher spin symmetries have been also studied, but their form is quite complicated. The continual Toda equation also exhibits a global $U(1)$ symmetry

$$
\delta \Psi=\epsilon \partial_{3} \Psi
$$

where $\epsilon$ is now taken to be independent of all space time variables. These symmetries, by construction, transform any self-dual gravitational background with $\Delta S_{-}>0$ into another, while preserving self-duality. They can certainly be employed to induce a mapping between non-trivial string backgrounds by intertwining them with T-duality. From this point of view one might suspect that the S-duality, if it always preserves the self-duality of the original metric, will be identified with a subgroup of the infinite dimensional symmetry group of the continual Toda theory. However, as we will see shortly this turns out not to be the case and we discover an anomaly for $\Delta S_{-} \neq 0$.

The simplest set of solutions of the continual Toda field equation can be obtained by making the ansatz

$$
e^{\Psi\left(x^{1}, x^{2}, x^{3}\right)}=\left(\alpha\left(x^{3}\right)^{2}+\beta x^{3}+\gamma\right) e^{\varphi\left(x^{1}, x^{2}\right)} .
$$

It is straightforward to verify that this ansatz will provide a class of solutions if $\varphi\left(x^{1}, x^{2}\right)$ satisfies the Liouville equation with coupling constant proportional to $\alpha$. The most general solution of the continual Toda equation has been constructed in the literature (see [18] and references therein), but it will not be needed for the present purposes. The solution

$$
e^{\Psi}=\frac{\left(x^{3}\right)^{2}-a^{2}}{2\left(1+\frac{1}{8}\left(\left(x^{1}\right)^{2}+\left(x^{2}\right)^{2}\right)\right)^{2}}, \quad\left(x^{3}\right)^{2} \geq a^{2}
$$

provides an alternative description of the Eguchi-Hanson instanton with respect to its second (rotational) Killing symmetry. The solution

$$
\Psi=\log x^{3}
$$

corresponds to the case

$$
d s^{2}=\left(d x^{1}\right)^{2}+\left(d x^{2}\right)^{2}+\frac{1}{x^{3}}\left(d x^{3}\right)^{2}+x^{3}(d \tau)^{2},
$$

which gives rise to the trivial flat metric after performing the coordinate transformation

$$
w_{1}=2 \sqrt{x^{3}} \cos (\tau / 2), \quad w_{2}=2 \sqrt{x^{3}} \sin (\tau / 2)
$$

The important point that needs to be made for rotational Killing symmetries is that the $\mathrm{T}-\mathrm{S}-\mathrm{T}$ transformation is anomalous, thus breaking the self-duality of the original

${ }^{\ddagger}$ The Eguchi-Hanson instanton has also a translational Killing symmetry in the sense that the corresponding $\Delta S_{-}=0$. This can also be seen here from the special combination of the $x^{1}$ and $x^{2}$ coordinates that appear in eq. (35). 
metric, even though the resulting pure gravitational background is Ricci flat. This is a classical obstruction that has nothing to do with quantum corrections. The metric maintains its rotational symmetry by performing the sequence of $\mathrm{T}-\mathrm{S}-\mathrm{T}$ transformations, but there is no coordinate system of the form $(28),(29)$ for a new scalar function $\Psi^{\prime}$, as it would have been required by the theorem [13] for rotationally invariant self-dual metrics. We will see shortly that this problem, which can be observed in Euclidean gravity without ever employing the string viewpoint, has a natural supersymmetric explanation in the lowest order string effective theory.

The aparent incompatibility between S-duality and self-duality (and hence $N=$ 4 superconformal symmetry) can be easily verified by performing the T-S-T loop of operations to the flat metric associated with the solution (36). Indeed, the metric we find at the end of the calculation is

$d s^{2}=\frac{x^{3}}{1-\left(C x^{3}\right)^{2}}\left(d \tau+C\left(x^{2} d x^{1}-x^{1} d x^{2}\right)\right)^{2}+\frac{1-\left(C x^{3}\right)^{2}}{x^{3}}\left(x^{3}\left(\left(d x^{1}\right)^{2}+\left(d x^{2}\right)^{2}\right)+\left(d x^{3}\right)^{2}\right)$

using for simpicity only elements of the Ehlers transform. It can be verified that for $C=0$ we obtain the original flat metric, while for any $C \neq 0$ the resulting metric is not self-dual (nor anti self-dual). Similarly, if we had applied the same method to the Eguchi-Hanson instanton with respect to its rotational symmetry, a violation of self-duality would have also been observed. Of course, as it was pointed out earlier, S-duality would not lead to such a violation if the $\mathrm{T}-\mathrm{S}-\mathrm{T}$ transformation were performed using the translational Killing symmetry of the same solution. Therefore, on general grounds, if there is an anomaly in $\Delta S_{-}$, the $\mathrm{S}$-duality appears to be incompatible with $N=4$ superconformal string theory. Instead, the symmetry which seems compatible with self-duality in this case is $W_{\infty}$, at least in the pure gravitational sector of the string effective theory.

This result points to a problem which actually turns out to be related with the $\mathrm{T}$-duality used to perform the combined $\mathrm{T}-\mathrm{S}-\mathrm{T}$ transformation. The reason is that for rotational Killing symmetries, unlike for the translational ones, T-duality does not preserve the (standard) space time supersymmetry. The obstruction can be easily seen by considering the supersymmetric variation of the dilatino field $\lambda$. Remember that in the heterotic string context we are looking for bosonic ground states of a supersymmetric theory. The variation $\delta \lambda=0$ implies in four dimensions that the dilaton and the antisymmetric tensor fields have to satisfy in the $\sigma$-model frame the consistency condition

$$
\frac{1}{2} H_{\mu \nu \rho}=\mp \sqrt{\operatorname{det} G} \epsilon_{\mu \nu \rho}^{\sigma} \partial_{\sigma} \Phi
$$

at each stage of the duality transformations. Since we have started from pure gravitational self-dual metrics, rather than anti self-dual, the appropriate sign in the equation above is minus. Taking into account eq. (7), it is straightforward to verify that the supersymmetric condition for the dilatino field will be valid in the $\mathrm{T}$-dual background only if $S_{-}$is constant and hence $\Delta S_{-}=0$. Space time supersymmetry can not be preserved under T-duality for Killing vector fields with $\Delta S_{-}>0$. The subsequent application of the $\mathrm{S}$ and $\mathrm{T}$ transformations can only make things worse. 
Supersymmetric T-duality transformations can be safely performed only with respect to translational Killing symmetries. We have already seen that even the simplest space time backgrounds, like the flat space and the Eguchi-Hanson instanton, have the potential for exhibiting an anomalous supersymmetric behaviour under T-duality when a rotational Killing symmetry is used. Solutions with more than one Killing symmetry are in practice much easier to construct. It is worth emphasing, however, that solutions with rotational Killing symmetries usually admit a translational Killing symmetry as well, which can be used safely. The reason is that if a space time admits two Killing symmetries, both of them rotational, it will not be a generic real Euclidean solution of the self-duality conditions [13]. The requirement of reality will exclude this possibility if the two rotational Killing vectors $K_{1}$ and $K_{2}$ are assumed to form a closed algebra, i.e.,

$$
\left[K_{1}, K_{2}\right]=\alpha K_{1}+\beta K_{2}
$$

A way out is thus provided by the existence of an extra translational Killing vector field leading to an $S U(2)$ symmetry algebra. The multi-center Gibbons-Hawking instantons with $n>2$ have only one Killing symmetry which is translational and hence safe under T-duality.

There are no real self-dual Euclidean solutions known to this date which exhibit only one rotational Killing symmetry with no other symmetries of either type. New series of well-behaved gravitational instatons can only be obtained by investigating carefully the space time interpretation of the general solution of the continual Toda equation [18]. If purely rotational instantons exist, $\mathrm{T}$-duality will never be compatible with their supersymmetry. The problem we are addressing here has also some relation with the nonabelian duality transformations of gravitational string backgrounds [19]. The presence of a rotational Killing vector field is a common element in both cases. For this reason we think that space time supersymmetry can not be preserved by non-abelian duality. We hope to return to this issue elsewhere taking into account gravitational backgrounds with non-trivial axion and dilaton fields as well.

We note finally that if both $\mathrm{S}$ and $\mathrm{T}$ are symmetries of a certain class of 4-dim string backgrounds with one Killing symmetry and with non-trivial antisymmetric tensor and dilaton fields, there will be two independent $S L(2, R)$ symmetries of the corresponding effective theory, namely $\mathrm{S}$ and $\mathrm{T}-\mathrm{S}-\mathrm{T}$. It seems that in this way the combination of the $\mathrm{S}$ and $\mathrm{T}$ duality transformations enlarges the symmetry group of the theory to $O(2,2)$. This result is also expected from the reduced form of the 4 -dim effective action. The reduction of pure gravity from four to three dimensions is formulated as an $S L(2, R) / U(1)$ $\sigma$-model coupled to 3-dim gravity, while the axion-dilaton system provides a second $S L(2, R) / U(1) \sigma$ model. A similar proposal was made recently for the compactification of the heterotic string on a seven dimensional torus, where the T-duality group $O(7,23)$ and S-duality combine by intertwing into $O(8,24)[20]$. Going one dimension lower we expect the string duality group to become an infinite dimensional discrete subgroup of $\hat{O}(8,24)$, in analogy with the infinite dimensional Geroch group $\hat{O}(2,2)$ of 4 -dim strings with two Killing symmetries [21]. The intertwining of the $\mathrm{S}$ and $\mathrm{T}$ transformations 
produces infinitely many new transformations in two dimensions. The implications of such huge symmetries to the spectrum of the eight dimensional compactification of the heterotic string, are also under consideration [22].

In summary, we found that $\Delta S_{-}$is an index for the Killing symmetry that determines whether T-duality preserves supersymmetry. An obstruction was observed for $\Delta S_{-} \neq 0$. Its generalization to more arbitrary backgrounds is an interesting problem. We also gave a reformulation of the Ehlers-Geroch hidden symmetry of the reduced theory of 4-dim gravity in terms of the S-duality transformations of the lowest order string effective theory. It was considered as providing a space time interpretation of S-duality. The simplest example was the transformation of the flat space to the Taub-NUT background.

\section{Acknowledgments}

I am grateful to Amit Giveon and Elias Kiritsis for collaboration on various aspects of the present work and for sharing their views on the problem of string duality symmetries. I have also benefited from conversations with Louis Alvarez-Gaume, Massimo Bianchi, Sergio Ferrara, Bernard Julia, Costas Kounnas and Dieter Lust.

\section{REFERENCES}

1. A. Font, L. Ibanez, D. Lust and F. Quevedo, Phys. Lett. B249 (1990) 35.

2. J.H. Schwarz and A. Sen, Phys. Lett. B312 (1993) 105; J.H. Schwarz, "Does String Theory Have a Duality Symmetry Relating Weak and Strong Coupling?", preprint CALT-68-1879, hep-th/9307121, July 1993; A. Sen, "Strong-Weak Coupling Duality in Four Dimensional String Theory", preprint TIFR/TH/94-03, hepth/9402002, February 1994.

3. J.W. van Holten, "Supersymmetry and the Geometry of Taub-NUT", preprint NIKHEF-H/94-29, hep-th/9409139, September 1994.

4. A. Dabholkar, G. Gibbons, J.A. Harvey and F. Ruiz-Ruiz, Nucl. Phys. B340 (1990) 33; A. Strominger, Nucl. Phys. B343 (1990) 167; C. Callan, J.A. Harvey and A. Strominger, Nucl. Phys. B359 (1991) 611, B367 (1991) 60.

5. S.J. Rey, Phys. Rev. D43 (1991) 526; M. Duff and J.X. Lu, Nucl. Phys. B354 (1991) 141; R. Khuri, Nucl. Phys. B387 (1992) 315; E. Kiritsis, C. Kounnas and D. Lust, Int. J. Mod. Phys. A $\underline{\text { 9 }}$ (1994) 1361.

6. M. Bianchi, F. Fucito, G.C. Rossi and M. Martellini, "ALE Instantons in String Effective Theory", preprint ROM2F-94-17, hep-th/9409037, September 1994.

7. I. Bakas and E. Kiritsis, work in progress. 
8. E. Fradkin and A. Tseytlin, Nucl. Phys. B261 (1985) 1; C. Callan, D. Friedan, E. Martinec and M. Perry, Nucl. Phys. B262 (1985) 593.

9. A. Giveon, M. Porrati and E. Rabinovici, "Target Space Duality in String Theory", preprint RI-1-94, hep-th/9401139, January 1994.

10. J. Ehlers, in "Les Theories Relativistes de la Gravitation", CNRS, Paris (1959); R. Geroch, J. Math. Phys. 12 (1971) 918.

11. G. Gibbons and S. Hawking, Commun. Math. Phys. $\underline{66}$ (1979) 291.

12. K.P. Tod and R.S. Ward, Proc. R. Soc. Lond. A368 (1979) 411.

13. C.P. Boyer and J.D. Finley, J. Math. Phys. 23 (1982) 1126; J.D. Gegenberg and A. Das, Gen. Rel. Grav. 16 (1984) 817.

14. T. Eguchi, P.B. Gilkey and A.J. Hanson, Phys. Rep. $\underline{66}$ (1980) 213.

15. N. Hitchin, Math. Proc. Camb. Phil. Soc. $\underline{85}$ (1979) 465; P.B. Kronheimer, J. Diff. Geom. 29 (1989) 665, 685.

16. A. Giveon and E. Kiritsis, Nucl. Phys. B411 (1994) 487; E. Kiritsis and C. Kounnas, Phys. Lett. B331 (1994) 51.

17. I. Bakas, in the "Proceedings of the Trieste Conference on Supermembranes and Physics in 2+1 Dimensions", eds. M. Duff, C. Pope and E. Sezgin, World Scientific, Singapore (1990); Q.H. Park, Phys. Lett. B236 (1990) 429; J. Avan, Phys. Lett.

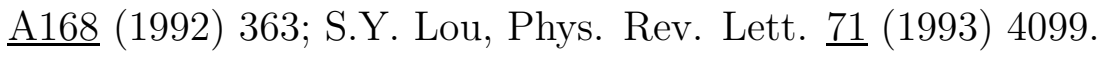

18. M.V. Saveliev, Theor. Math. Phys. $\underline{92}$ (1992) 457; M.V. Saveliev and S.A. Savelieva, Phys. Lett. B313 (1993) 55.

19. X. de la Ossa and F. Quevedo, Nucl. Phys. B403 (1993) 377; A. Giveon and M. Rocek, Nucl. Phys. B421 (1994) 173; E. Alvarez, L. Alvarez-Gaume and Y. Lozano, "On Non-Abelian Duality", preprint CERN-TH.7204/94, hep-th/9403155, March 1994.

20. A. Sen, "Strong-Weak Coupling Duality in Three Dimensional String Theory", preprint TIFR/TH-94-19, hep-th/9408083, August 1994.

21. I. Bakas, "O(2,2) Transformations and the String Geroch Group", preprint CERNTH.7144/94, hep-th/9402016, February 1994.

22. I. Bakas and A. Giveon, work in progress. 